\title{
Province-Wide Healthcare System Integration Challenge Being Solved in Alberta
}

Myrna Francis

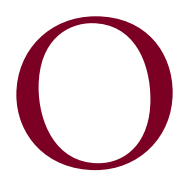

ver the past 10 years, Alberta has pioneered the development of a province-wide electronic health record (EHR). As a result, a number of the building blocks are already operational - for example, patient and provider registries, a pharmaceutical information network, a laboratory test results history system and electronic medical record systems are in many physicians' offices. However, because these are stand-alone applications based on local databases, the full breadth of cross-province patient information cannot be accessed. The big challenge facing Alberta, therefore, is interconnecting these systems and information repositories so that data can be exchanged seamlessly. This would give authorized physicians, nurses, allied healthcare workers, managers and administrators a single point of electronic access to a patient's complete medical profile and history province-wide.

Work is already under way to interconnect disparate data systems and information repositories within Alberta's individual health regions, such as the Calgary Health Region (CHR). Here, a regional integration engine provides a basic layer of operational connectivity, tying together various healthcare facilities (e.g., hospitals, laboratories and clinics), applications, patient indices and data repositories within the region. The result is an EHRlike capability at the regional level. A similar integration structure exists in the Capital Health Region based in Edmonton, and such structures are also being built within the Alberta Cancer Board and the Regional Shared Health Information Program, a partnership among the seven remaining Alberta healthcare regions, covering $92 \%$ of the province's vast geography. "We are learning a lot of lessons from these regional initiatives," says Linda Miller, assistant deputy minister, Alberta Health and Wellness (AHW). "They are generating a lot of interest - especially among physicians - as people begin to understand what an EHR really is and the value of the data beyond the bounds of any one healthcare organization."

\section{Regions Set Stage for Provincial Integration}

AHW's mandate, however, is to go beyond what the regions have accomplished, to create a provincial-level EHR system. This requires a set of services that physicians and other healthcare workers would use and the data that these services and users would access. The EHR viewer and Laboratory Repository access services are being developed by Capital Health on behalf of AHW, while the integration and interoperability services are being handled by CHR in what is being called the provincial Health Information Exchange ( $\mathrm{pHIE}$ ) project, also sponsored by AHW. CHR has engaged information technology (IT) and business process services provider CGI to design, build, deploy and operate the pHIE on its behalf.

\section{"Work is already underway to interconnect disparate data systems and information reposi-} tories within Alberta's individual health regions."

Loosely analogous to an electrical outlet into which appliances are plugged to access power, the pHIE is the interconnection point into which EHR components - applications, repositories, viewers and so on - can be plugged to allow access to, and sharing of, a common set of data, specifically patient 
information. AHW's decision to have CHR handle the pHIE not only puts the work closer to the "ground" - closer to the actual physicians and other healthcare workers who know best what is needed and who must ultimately be willing to adopt the system - it also taps into the experience that CHR gained in creating its own regional integration capability. According to Linda Miller, CHR has been grappling with this whole system connectivity and message brokering challenge for some time now and has developed its own regional integration engine; AHW wanted to capitalize on that experience.

Since the data for Alberta's EHR system will come from hundreds of different provincial and regional sources and healthcare systems, where data exists in many different formats, the role of the pHIE initially is to provide basic integration and interoperability. At this fundamental level, the job of the pHIE is to interconnect all the different systems yet abstract them from each other so that each system can continue evolving at its own rate. This basic integration task is made somewhat easier by the existence of the four regional integration engines. The pHIE needs only to connect to these regional integration hubs - bringing together the integration engines - rather than having to connect separately to every system and domain repository within each region. The pHIE will also act as the single interface to provincial registries, as well as to other systems such as electronic medical record systems.

While starting out initially as a message broker between differing systems, the pHIE will ultimately do much more. It will provide a common services and communications "infostructure" layer that is consistent with Canada Health Infoway's architectural definition of the Health Information Access Layer.

\section{"While healthcare in the past typically consisted of singular events - one visit to a general practi- tioner or hospital and you're done - we are living and staying active much longer. People now have an expectation of 'continuity of care' over their lifetime."}

\section{Best-Practice Care Delivery}

The pHIE will play a role at the highest level of the connectivity hierarchy, bridging differences in healthcare processes or workflows that may exist between various systems, and in applying rules to help ensure the delivery of best-care practices. "While healthcare in the past typically consisted of singular events - one visit to a general practitioner or hospital and you're done - we are living and staying active much longer. People now have an expectation of 'continuity of care' over their lifetime," states Bill Trafford, vice-president, Advanced Technology, CHR.
Fundamental to the continuity-of-care concept is the requirement that healthcare providers follow a best-practices care plan regardless of treatment location. It is the inconsistent delivery of care - two physicians in two jurisdictions treating the same patient differently - that causes churning in the healthcare system, drives up the cost and sometimes even puts patients at risk.

Since the pHIE serves as a cross-jurisdictional hub for the integration of systems and information province-wide, it will become a critical enabler in helping Alberta address the dual challenge of delivering best-practices care as part of a continuity of care that spans the province. With the help of physician experts to ensure the results are based on "true clinical knowledge," CHR has already created best-practice care plans for more than 1,400 diseases.

According to Bill Trafford these care plans constitute the "business rules" of our healthcare industry, and these clinical rules are being embedded into the pHIE so that the systems can help every physician, nurse and allied healthcare worker deliver the same best-practices care plans and maintain continuity of care throughout the province.

\section{E-health Platform for the Future}

Like the electricity grid, the pHIE is, in essence, just a utility that does nothing until applications are plugged into it. The first will be Alberta's EHR services; but once all the systems and data repositories are interconnected, the pHIE will become an integration and interoperability platform that will support a vast array of e-health applications - wait list systems, electronic referral systems, scheduling systems and many others as yet unknown.

The Internet went this same route, starting with basic browsing, which is a simple, read-only transaction. Once the global networking infrastructure was in place and people started to see what it could do, the use of the Internet exploded virtually overnight, with ever-more-complex, fully interactive and highly beneficial applications being added every day.

The connectivity and integration challenge has been met, and solved, in a number of industries as well, most notably the banking industry. While healthcare presents some unique challenges, these too are on the verge of being solved; and AHW recognizes that while building an EHR solution, it is also establishing an integration platform for a host of future e-health initiatives.

\section{About the Author}

Myrna Francis, PhD, is the vice-president of Global Marketing for the health industry at CGI, with responsibility for supporting the IT and e-health needs of government health departments and healthcare providers. 
